\title{
CLUSTERING-BASED MARKET SEGMENTATION USING NEURAL NETWORK MODELS
}

\author{
Harald Hruschka, Universität Regensburg \\ Martin Natter, Institut für Höhere Studien, Wien
}

Most segmentation studies proceed in two steps, determining segments in the first step and looking for discriminating characteristics (segment descriptors) in the second step. Data-analytic methods usually applied in these steps are cluster analysis and discriminant analysis techniques, respectively. Artificial neurål networks represent alternatives to better known statistical techniques. Certain types of artificial neural networks are closely related to well-known statistical methods. Principal components analysis, for example, is a special case of certain artificial neural network models. Therefore use of neural network models in market segmentation seems to be justified considering their greater generality.

In this paper two types of feedforward neural networks with logistic activation functions are specified. Models of the first type determine segments on the basis of segmentation criteria. These classification models are feedforward neural networks using segmentation criteria both as input variables and output variables. Between input and output variables we put a layer of hidden units. The task of the classification and discrimination models is more encompassing. Models of this type simultaneously form segments and discriminate between these segments on the basis of additional segment descriptors. Parameters of all models are estimated by an extended version of backpropagation.

The main marketing-related objective of the study presented here is to gain a general understanding of usage patterns of the main brands of a non-durable product class. Therefore usages of brands in different situations are chosen as segmentation criteria. The respondents are a representative random sample of 1007 persons. After deletion of incorrect data and limitation to the more frequent brands and situations, the data base consists of 854 respondents.

Using nonlinear neural networks for clustering-based segmentation lead to lower error measures compared to their linear counterparts. Interpretation of model results is emphasized in view of its great importance in Marketing applications. 